\title{
Automated tru-cut imaging-guided core needle biopsy of canine orbital neoplasia. A prospective feasibility study
}

\author{
A. Cirla ${ }^{1, *}$, M. Rondena ${ }^{2}$ and G. Bertolini ${ }^{1}$ \\ ${ }^{1}$ San Marco Veterinary Clinic, via Sorio 114/c - 35141 Padova, Italy \\ ${ }^{2}$ San Marco Veterinary Laboratory, via Sorio 114/c - 35141 Padova, Italy
}

\begin{abstract}
The purpose of this study was to evaluate the diagnostic value of imaging-guided core needle biopsy for canine orbital mass diagnosis. A second excisional biopsy obtained during surgery or necropsy was used as the reference standard. A prospective feasibility study was conducted in 23 canine orbital masses at a single centre. A complete ophthalmic examination was always followed by orbital ultrasound and computed tomography (CT) examination of the head. All masses were sampled with the patient still on the CT table using ultrasound (US) guided automatic tru-cut device. The most suitable sampling approach to the orbit was chosen each time based on the CT image analysis. One of the following different approaches was used: trans-orbital, trans-conjunctival or trans-masseteric. In all cases, the imaging-guided biopsy provided a sufficient amount of tissue for the histopathological diagnosis, which concurred with the biopsies obtained using the excisional technique. CT examination was essential for morphological diagnosis and provided detailed topographic information that allowed us to choose the safest orbital approach for the biopsy. US guided automatic tru-cut biopsy based on CT images, performed with patient still on the CT table, resulted in a minimally invasive, relatively easy, and accurate diagnostic procedure in dogs with orbital masses.
\end{abstract}

Keywords: Computed tomography, Core-needle biopsy, Dogs, Orbital mass, Ultrasound.

\section{Introduction}

A number of neoplasias have been reported to affect the orbit or retrobulbar space in dogs (Hendrix and Gelatt, 2000; Kato et al., 2012; Spiess and Pot, 2013). These neoplasias can be primary or secondary tumors reaching the orbital region via either metastasis or extension from adjacent sites (Attali-Soussay et al., 2001; Van der Woerdt, 2008). Orbital neoplasia can be easily detected on advanced imaging examinations in many circumstances. It may be found incidentally on computed tomography (CT) or magnetic resonance imaging (MRI) examinations of the skull, performed for other non-ophthalmological diseases (Armour et al., 2011; Boland et al., 2013). More often, however, the lesion may be looked for directly with imaging in those patients with ophthalmological signs suspected for an orbital lesion. With few exceptions, the majority of orbital diseases are space-occupying lesions leading to characteristic clinical signs such as exophthalmos and orbital or periorbital pain (Featherstone and Heinrich, 2013). Orbital neoplasia should be differentiated from inflammatory conditions such as orbital abscess or orbital cellulitis. Orbital CT has been reported as a highly specific imaging test for differentiating inflammatory and neoplastic conditions in small animals (Boroffka et al., 2007; Wang et al., 2009; Armour et al., 2011; Lederer et al., 2015).

Given the wide variety of diseases that can be encountered, advanced imaging modalities play a major role in the diagnostic and staging assessment of the lesion, guiding therapeutic decisions and monitoring or follow-up of treatment (Penninck et al., 2001; Collins et al., 2013). CT, MRI (Boroffka and Voorhout, 1999; Armour et al., 2011; Lederer et al., 2015) and ultrasound (US) have been demonstrated to be accurate in evaluating orbital disease (Mason et al., 2001; Boroffka et al., 2007). In addition, US has been demonstrated to be particularly useful in guiding interventional procedures throughout the body both in veterinary and in human patients (Phillips and Schneider, 1981; Gupta et al., 1999; Constantin et al., 2010; Orlandi et al., 2013; Spiess and Pot, 2013). However, imaging alone is not sufficient for a definitive diagnosis (Boroffka and Voorhout, 1999; Boroffka et al., 2007). To determine the nature of the mass and choose an appropriate treatment, pathological investigations remain necessary (Hendrix and Gelatt, 2000; Attali-Soussay et al., 2001). Invasive diagnostic modalities in orbital neoplasia include excisional and incisional biopsy (open biopsy). While these methods provide sufficient histological tissue, they require high morbidity surgical intervention (Slatter and Abdelbaki, 1979; Gilger et al., 1994; Boston, 2010; Hakannsson and Hakannsson, 2010; Gelatt and Withley, 2011; Spiess and Pot, 2013). In contrast, fine needle aspiration biopsy is effective and sometimes may be acceptable in ocular oncology (Tani et al., 2006; Agrawi et al., 2013; Nair and Sankar, 2014). Imaging-guided core needle 
biopsy is often performed in oncology, and unlike fine needle aspiration biopsy, it can provide sufficient tissue sample for histology (Ballo and Sneige, 1996; Yarovoy et al., 2013). To be effective, an imaging-guided core needle biopsy technique requires precise localization and documentation of the needle inside the target lesion, especially in cases of deep retrobulbar lesions. To date, few articles in veterinary literature describe a US-CT multimodality approach for orbital mass sampling for morphologic examination (LeCouteur et al., 1982; Boroffka and Voorhout, 1999; Boroffka et al., 2007; Lederer et al., 2015). In this study, we investigate the feasibility and accuracy of imagingguided core needle biopsy in a population of dogs with orbital masses, using a US-CT combined system and different biopsy approaches.

\section{Material and Methods}

\section{Animals}

Subjects for this prospective feasibility study were enrolled from dogs referred to the ophthalmology service of the San Marco Veterinary Clinic during a 2-year period, from April 2012 through April 2014. Dogs were included in the study based on a presumptive clinical and imaging diagnosis of orbital neoplasia in at least one orbit.

\section{Ophthalmic examination}

Each patient received physical and complete ophthalmic examinations. All the ophthalmic examinations were performed by the same ophthalmologist (AC). Complete ophthalmic examination included neuroophthalmic examination, slit-lamp biomicroscopy (SL-15, Kowa Company Ltd, Tokyo, Japan), and indirect ophthalmoscopy (Heine Omega 500 and Heine 30D lens; Heine Optotechnik Inc, Herrsching, Germany). Schirmer tear test I (Schirmer Tear Test, Schering-Plough Animal Health, Union, NJ), retention of corneal sodium fluorescin dye (HS Haag-Streit International fluorescein, Switzerland) and intraocular pressure estimation (TonoPen Vet, Reichert Inc., Depew, NY) were performed.

The orbital mass was suspected during ophthalmic examination and confirmation was obtained by orbital ultrasound. To further characterize the mass, staging, and therapeutic planning, all dogs underwent 16-multidetector-row CT (16-MDCT, Lightspeed 16, GE Healthcare, Milan, Italy) or 128-dual source CT (SOMATOM Definition Flash, Siemens Healthcare, Forchheim, Germany). Owner consent allowed sedation and anesthesia for CT and CT-US combined biopsy procedures. Complete hemato-biochemical work-up (complete blood count, biochemical profile, serum protein electrophoresis, and coagulation tests), and urinalysis were performed for all patients prior to the procedures.

\section{MDCT scanning protocol}

Food and water were withheld from dogs overnight prior to CT procedures. A 14-16 GA intravenous catheter was placed in the right or left cephalic vein for fluid administration throughout the anaesthetic period. All patients were premedicated using methadone (0.2 mg/kg IM; Semfortan, Dechra Pharmaceuticals) and dexmedetomidine (2 mg/kg IM; Dexdomitor, Orino Pharma). General anesthesia was inducted with propofol (5 mg/kg IV; Vetofol, Esteve), and mantained with isoflurane (IsoFlo; Halocarbon Laboratories) in oxygen. Multidetector CT scans of the head were obtained with patient in sternal recumbency on the CT table with open mouth. Acquisition parameters were as follow: helical modality, detector configuration $16 \times 0.625$, pitch $0.562: 1$, and $0.7 \mathrm{~s}$ gantry rotation. Dose parameters were $120 \mathrm{kVp}$ and $210 \mathrm{mAs}$. Images were reconstructed using both bone and standard algorithm (non-enhancing-non-smoothing reconstruction algorithm) with $512 \times 512$ matrix size and $50 \%$ overlap section thickness. Non-contrast and contrast scans were always obtained. For enhanced scans, iodixanol (320 mg I/mL), an iodinated, non-ionic, iso-osmolar contrast medium, was injected at $37^{\circ} \mathrm{C}$ into a cephalic vein $(640 \mathrm{mg} \mathrm{I} / \mathrm{kg})$ through the intravenous catheter. A uniphasic injection $(3-5 \mathrm{~mL} / \mathrm{s})$ of the contrast medium was made, followed by a saline flush at same injection rate via a dual-syringe injector system (Medrad, Stellant CT Injection System). Multidetector CT data were reviewed directly at the CT-console (GB). Multiplanar reformation (MPR) post-processing techniques of both unenhanced and enhanced series were used to analyse site, shape, and appearance of the mass. Based on previously established imaging criteria (Boroffka et al., 2007; Lederer et al., 2015), orbital masses showing CT characteristics consistent with abscess or post-traumatic orbital hematoma were not sampled by tru-cut and instead excluded from this study.

\section{Technique, safety and diagnostic assessment of orbital biopsies}

The safest biopsy approach was based on the topography of the mass, as seen on orthogonal and multi-oblique CT-planes (Fig. 1). Care was taken to preserve neurovascular structures and regional nerves, in order to avoid fluid-suprafluid areas of the mass, interpretable as necrotic or hemorrhagic in nature. The cutting-needle US-guided procedure was performed immediately after the CT scan with the patient still under general anesthesia on the CT exam table. All biopsies were performed with use of a real time US system (Logiq C5 Premium, GE Healthcare, Milan, Italy) with a 10 or $12-\mathrm{Mhz}$ transducer. US guidance was obtained using either a linear or mini-convex probe according to the lesion location. Based on CT characteristics, one of the following three approaches was performed: trans-orbital, trans-conjunctival or trans-masseteric (Fig. 2). The path of the needle was monitored by means of continuous US visualization. By 


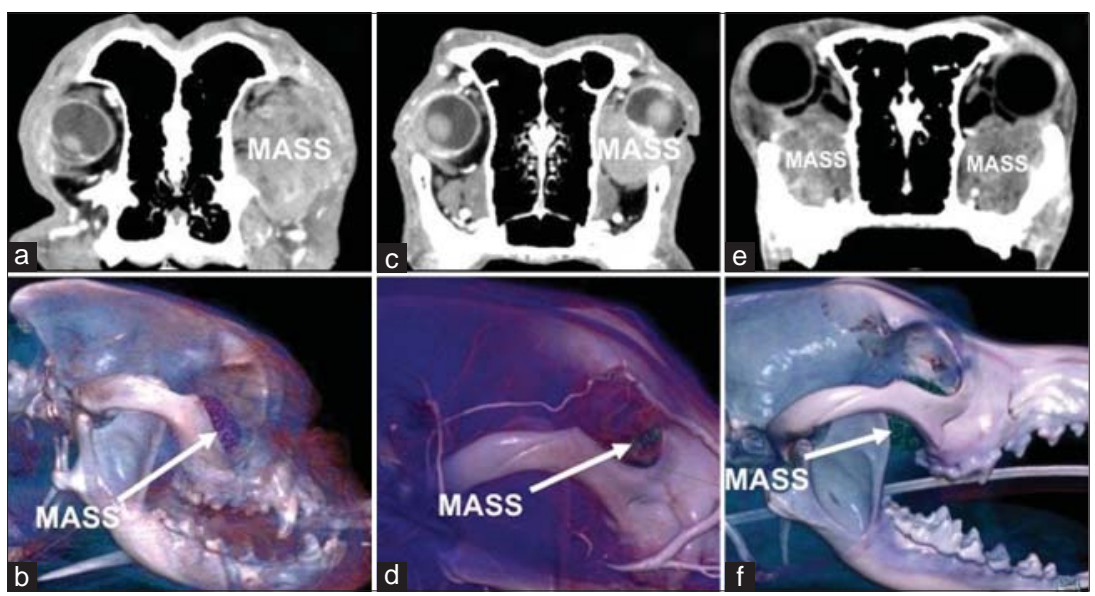

Fig. 1. Images from MDCT examinations of the skull of three different dogs with orbital masses. (a and b) Transverse and 3D volume rendered (right lateral view of the skull) images of a Boxer (case 4) having a sarcoma in the left orbit, postero-inferior to the globe. (c and d) are transverse and right lateral views of 3D volumes rendered images respectively in an American Cocker Spaniel (case 11) with a left-sided orbital rhabdomyosarcoma located anterior to the globe. (e and f) are transverse and right-lateral 3D volume rendered images of a Border Collie (case 3 ) with bilateral orbital masses located postero-ventrally the eyeballs (B-cell lymphoma).

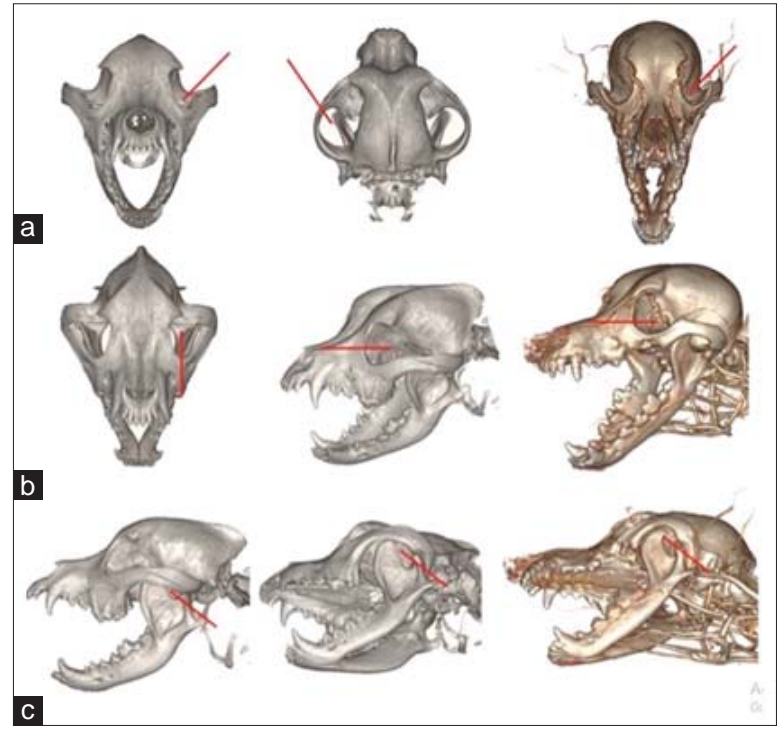

Fig. 2. (a) Trans-orbital approach to the posterior orbital space (supero and infero-lateral). With the patient in sternal recumbency, the biopsy needle is inserted just dorsal to the zygomatic arch and guided in a $45^{\circ}$ oblique ventral direction. (b) Trans-conjunctival approach to the anterior orbital space (ventro-lateral and medial). With the patient in sternal recumbency, the biopsy needle is placed on the edge of the lacrimal bone. (c) Trans-masseteric approach to the posterior orbital space and to its floor. With the patient in lateral recumbency with open mouth, the biopsy needle is positioned in the space between the mandibular coronoid process and the zygomatic temporal process.

using an aseptic technique, all the masses were sampled with an automatic high-speed core biopsy system (Bard MagnumBiopsy System, Bard Peripheral Vascular, Inc., Tempe, AZ) with sterile disposable needle (14G, $16 \mathrm{G})$, and a depth of penetration ranging from 15 to
$22 \mathrm{~mm}$. An adequate sterile field around the affected eye was set and, if necessary, a $2 \mathrm{~mm}$ skin incision was made to facilitate the core needle insertion. The needle was inserted into the orbit slowly during puncture while controlling the resistance and observing the eye globe condition. The orbital mass was punctured one time. After the procedure, the puncture site was manually compressed for 2-5 minutes and a compressive bandage and ice were applied over the relevant eye when needed. All the patients were kept under observation for 1 hour, an Elizabethan collar prevented self-trauma at the biopsy site. Carprofen was given the first 24 hours post procedure (2 $\mathrm{mg} \backslash \mathrm{Kg}$ IV; Rimadyl, Pfizer Animal Health) to address pain and inflammation.

Methods that were used for determining safety of the procedure included: 1) biopsy site monitoring by CT scan immediately after the procedure 2) orbital US monitoring 1 and 2 hours, post-biopsy and prior to the discharge. 3) a 3 and 6-day clinical recheck.

Presence or absence of complications related to the biopsy procedure were recorded each time stated 1-3.

To assess the diagnostic performance of the tru-cut samples, an excisional biopsy was obtained from 11/17 patients during surgical procedures and 6/17 patients from necropsy representing the reference standard. The main reason for open biopsy after imaging-guided core needle biopsy was the surgical treatment. In case of necropsy, written informed consent was obtained from each owner. All the biopsy specimens obtained were fixed in $10 \%$ buffered isotonic formalin dehydratated with automated histological processor (Shandon Excelsior ES, Thermo Scientific, Fremont, CA) and paraffin embedded. Four micrometer-thick microtomic sections were then obtained and hematoxylin eosin stained for histologic evaluation (Fig. 3). 


\section{Immunohistochemistry (IHC)}

IHC was performed on lymphomas and rhabdomyosarcoma using ABC peroxidase method. Four micrometer-thick sections were rehydratated and endogenous peroxidase blocking were performed with immersing sections in H2O2-PBS solution for 45 minutes. Primary antibodies were applied for 18 hours at $4^{\circ} \mathrm{C}$ as reported in Table 1 . Secondary biotilated antibody (Vector $\AA$ ) was applied for 30 minutes at room temperature (RT). ABC complex (Vector $\AA$ ) was then applied for 30 minutes at RT. Signal was stained with IMMPACT Nova RED (Vector SK-4805) and sections were counterstained with hematoxylin, dehydratated in serial alcohol and xylene, then coverslide was mounted.

\section{Results}

Seventeen dogs (7 Mongrels, 1 Dobermann, 1 Rottweiler, 1 Border Collie, 1 Boxer, 1 Golden Retriever, 1 Dachshund, 1 American Cocker Spaniel, 1 Belgian Shepherd, 1 Miniature Poodle, 1 German
Shepherd), 9 males and 8 females met the inclusion criteria and were included in the study. The median age was 8.1 years (range from 1.1 to 13.8 years) and the median body weight was $17.7 \mathrm{~kg}$ (ranging from 6 to $48 \mathrm{~kg}$ ) (Table 2). Eleven/17 dogs presented with unilateral orbital neoplasia (4 right eye, 7 left eye) and in $6 / 17$ dogs the orbital disease was bilateral. A total of 23 orbital masses, having a maximum diameter between 25 and $38.5 \mathrm{~mm}$ were studied. Where bilateral both masses were sampled.

All the 23 core biopsies yielded adequate specimens for histologic analysis. Based on pathology results, 11 masses (monolateral) underwent to surgery and an excisional biopsy was obtained during the procedure. Twelve masses (bilateral) were not treated, due to poor prognosis. In such cases, upon owner request, the dogs were euthanatized and necropsy was performed. In all the tru-cut biopsies the histologic analysis concurred with the larger sample obtained through surgical
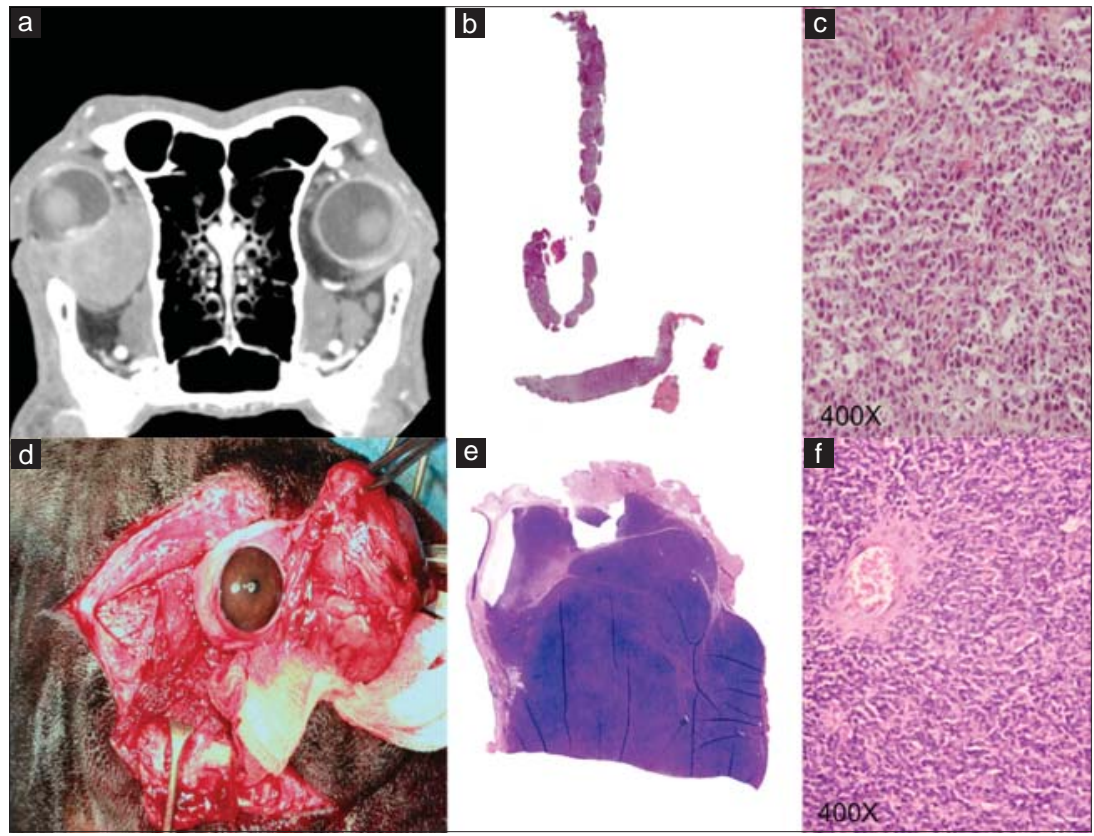

Fig. 3. Case 1. Dobermann. Anaplastic high grade STS in the right orbit anterior to the globe. (a) Transverse image from MDCT examination of the skull. (b and c) Tru-cut sample: subgross (b) and high magnification (c) of the histologically processed sample. (d) Intraoperative image of the same patient. (e and f) Surgical excisional biopsy: subgross (e) and high magnification (f) of the histologically processed sample. Note: similar quality in both sampling.

Table 1. IHC: Antibodies, source, dilution and antigen retrival.

\begin{tabular}{lllll}
\hline Primary antibody & Source & Clone & Dilution & Antigen retrival \\
\hline Anti CD3 & Dako & Policlonal & $1: 1000$ & Pepsin Solution Digest-All 3 (Novex) $10 \mathrm{~min}, 37^{\circ} \mathrm{C}$ \\
Anti CD20 & Thermo Scientific & Policlonal & $1: 1000$ & None \\
Anti Vimentin & Dako & V9 & $1: 1000$ & High Temperature pH $6.020 \mathrm{~min}, 95^{\circ} \mathrm{C}$ \\
Anti Actin & Dako & HHF35 & $1: 1000$ & High Temperature pH $6.020 \mathrm{~min}, 95^{\circ} \mathrm{C}$ \\
Anti Desmin & Dako & D33 & $1: 200$ & High Temperature pH $6.020 \mathrm{~min}, 95^{\circ} \mathrm{C}$ \\
Anti Myoglobin & Dako & Policlonal & $1: 200$ & High Temperature pH $6.020 \mathrm{~min}, 95^{\circ} \mathrm{C}$ \\
\hline
\end{tabular}


Table 2. Signalment and histological diagnosis.

\begin{tabular}{|c|c|c|c|c|c|}
\hline Case & Sex & $\begin{array}{c}\text { Age } \\
\text { (years) }\end{array}$ & Breed & Eye & Diagnosis \\
\hline 1 & M & 3 & Dobermann & OD & $\begin{array}{l}\text { Anaplastic high } \\
\text { grade STS }\end{array}$ \\
\hline 2 & $\mathrm{MN}$ & 10 & Rottweiler & OS & Metastatic melanoma \\
\hline 3 & M & 1.5 & Border Collie & $\mathrm{OU}$ & B-cell lymphoma \\
\hline 4 & $\mathrm{MN}$ & 8 & Boxer & OS & $\begin{array}{l}\text { Anaplastic high } \\
\text { grade STS }\end{array}$ \\
\hline 5 & FS & 13.3 & Dachshund & OS & MTB \\
\hline 6 & $\mathrm{MN}$ & 5.5 & Mongrel & $\mathrm{OU}$ & B-cell lymphoma \\
\hline 7 & $\mathrm{~F}$ & 9.6 & $\begin{array}{l}\text { Golden } \\
\text { Retriever }\end{array}$ & OD & $\begin{array}{l}\text { Anaplastic high } \\
\text { grade STS }\end{array}$ \\
\hline 8 & FS & 13.8 & Mongrel & OS & Fibrosarcoma \\
\hline 9 & FS & 10 & Mongrel & OU & B-cell lymphoma \\
\hline 10 & $\mathrm{MN}$ & 8.3 & Mongrel & OD & Fibrosarcoma \\
\hline 11 & M & 1.1 & $\begin{array}{l}\text { American } \\
\text { Cocker } \\
\text { Spaniel }\end{array}$ & OS & Rhabdomyosarcoma \\
\hline 12 & FS & 11 & $\begin{array}{l}\text { Belgian } \\
\text { Shepherd }\end{array}$ & OD & $\mathrm{SCC}$ \\
\hline 13 & M & 4.6 & Mongrel & OU & Fibrosarcoma \\
\hline 14 & $\mathrm{~F}$ & 7.5 & $\begin{array}{l}\text { Miniature } \\
\text { Poodle }\end{array}$ & OS & Fibrosarcoma \\
\hline 15 & M & 9 & $\begin{array}{l}\text { German } \\
\text { Shepherd }\end{array}$ & OS & MTB \\
\hline 16 & FS & 7.5 & Mongrel & $\mathrm{OU}$ & B-cell lymphoma \\
\hline 17 & FS & 12 & Mongrel & OU & B-cell lymphoma \\
\hline
\end{tabular}

F: Intact female, M: Intact male, SF: Spayed female, NM: Neutered male, OD: Right eye, OS: Left eye, OU: Both eyes, MTB: Multilobular tumor of bone, SCC: Squamous cell carcinoma, STS: Soft tissue sarcoma

excisional biopsy or during necropsy.

Twenty-three biopsies revealed a malignant process: anaplastic high grade soft tissue sarcoma $(n=3)$, fibrosarcoma $(n=5)$, rhabdomyosarcoma $(n=1)$, B-cell lymphoma $(n=10)$, metastatic melanoma $(n=1)$, squamous cell carcinoma $(n=1)$, multilobular tumor of bones $(n=2)$. One out of 17 patients $(5.88 \%)$ developed a localized hematoma at the puncture site that subsided spontaneously. No other early or later complications occurred.

\section{Discussion}

Surgical biopsy of space-occupying lesions of the orbit, particularly of those retrobulbar in location, presents substantial technical difficulties (Attaly-Soussay et al., 2001; Spiess and Pot, 2013). Papers reporting the use of CT guidance to perform biopsy of orbital masses are limited (Hendrix and Gelatt, 2000; Boroffka et al., 2007; Héranet al., 2014). Boroffka et al. (2007) made a comparison between helical CT and US guidance in 43 dogs affected by neoplastic and non-neoplastic orbital masses, reporting similar diagnostic value for both imaging techniques (Boroffka et al., 2007). A major disadvantage of CT over ultrasound was the lack or realtime visualisation during needle placement and biopsy. Our results demonstrated that imaging-guided core needle biopsy combining the two imaging modalities is a feasible, safe and effective procedure to perform histological biopsies in such a critical area of the body like the orbit. The procedure to obtain tissue is simple, minimally invasive and generally well tolerated by the patient. In contrast to fine needle aspiration biopsy, which is cytological, the aim of imaging-guided core needle biopsy is to obtain adequate tissue sample for histological diagnosis. Core needle biopsies are widely used in many oncological subspecialties as an effective alternative to open biopsy (Ortiz et al., 1996; Boland et al., 2013; Finger, 2014; Héran et al., 2014). Many recent studies, in human literature, report advantages of core biopsy over fine needle aspiration biopsy as oncological diagnostic procedure (Woodcock and Morgan, 1998; Orlandi et al., 2013). The automatedloaded tru-cut biopsy needles used in our study allowed tissue samples to be taken without tissue damage, because of a rapid cutting action and narrow diameter (14 and 16 gauge). The needle sizes used for the study resulted to be adequate for tissue sampling and safe for the orbital structures at the same time.

We had no cases of eye globe, optic nerve or orbital wall damage of clinical significance.

To prevent major complications, this procedure must be carefully planned by means of high quality CT data. An experienced ophthalmologist-radiologist team should perform the procedure under constant US visual control. This increases the accuracy and safety of the bioptic procedure. In our series of orbital neoplasia, we reported no inadequate specimens and final diagnosis was reached by the pathologist in $100 \%$ of cases. Small subcutaneous haematoma experienced in one dog resolved spontaneously in less than 6 days. It is important to know, that retrobulbar haematoma could represent a major complication with potentially irreversible visual sequelae. However, prompt diagnosis and immediate pharmacological therapy may prevent such an occurrence. Needle-track tumor seeding after fine needle aspiration biopsy/core needle biopsy is another possible complication as with any other type of needle biopsy, although literature reports are controversial (Diaz et al., 1991; Nyland et al., 2002; Vignoli et al., 2007; Brenner and Gordon, 2011; Klopfleisch et al., 2011). In this small group of patients, we did not see any evidence of tumor spread or recurrence at the site of biopsy. Furthermore, only in one out of seventeen dogs developed a localized hematoma at puncture site that subsided spontaneously. Results from our study suggests that the tru-cut imaging 
guided biopsy technique is a safe procedure. However, studies with a larger population should be performed to delineate the risk of metastatic spread and potential complications using this technique. All lesions included in our series were of $25 \mathrm{~mm}$ or larger. Thus, feasibility and accuracy of such a procedure in smaller lesions are yet to be demonstrated. Our case series includes 23 biopsies revealed a malignant process: anaplastic high grade soft tissue sarcoma $(n=3)$, fibrosarcoma $(n=5)$, rhabdomyosarcoma $(n=1)$, B-cell lymphoma $(n=10)$, metastatic melanoma $(n=1)$, squamous cell carcinoma $(n=1)$, and multilobular tumor of bones $(n=2)$. All bilateral neoplasias were related to the same oncologic disease (fibrosarcoma and B-cell lymphoma). In all these cases upon owner request the dogs were euthanatized due to the advanced stage of the diseases and the poor prognosis.

In conclusion, semi-automated imaging-guided core needle biopsy of orbital masses is a useful diagnostic technique in dogs. It provides a sufficient amount of tissue for histopathological analysis. The complications reported in our experience are minor; this procedure can really reduce the number of open biopsies and can be a useful supplement or alternative to fine needle aspiration biopsy in selected cases.

\section{Conflict of interest}

The authors declare that there is no conflict of interest

\section{References}

Agrawi, P., Dey, P. and Lal, A. 2013. Fine-Needle Aspiration Cytology of orbital and Eyelid Lesions. Diagn. Cytopathol. 41, 1000-1011.

Armour, M.D., Broome, M., Dell'Anna, G., Blades, N.J. and Esson, D.W. 2011. A review of orbital and intracranial magnetic resonance imaging in 79 canine and 13 feline patients (2004-2010). Vet. Ophthalmol. 14, 215-226.

Attali-Soussay, K., Jegou, J.P. and Clerc, B. 2001. Retrobulbar tumors in dogs and cats: 25 cases. Vet. Ophthalmol. 4, 19-27.

Ballo, M.S. and Sneige, N. 1996. Can core needle biopsy replace fine-needle aspiration cytology in the diagnosis of palpable breast carcinoma: A comparative study of 124 women. Cancer 78, 773-777.

Boland, L., Gomes, E., Payen, G., Bouvy, B. and Poncet, C. 2013. Zygomatic salivary gland diseases in the dog: three cases diagnosed by MRI. J. Am. Anim. Hosp. Assoc. 49, 333-337.

Boroffka, S.A. and Voorhout, G. 1999. Direct and reconstructed multiplanar computed tomography of the orbits of healthy dogs. Am. J. Vet. Res. 60, 1500-1507.

Boroffka, S.A., Verbruggen, A.M., Grinwis, G.C.M., Voorhout, G. and Barthez, P.Y. 2007. Assessment of ultrasonography and computed tomography for the evaluation of unilateral orbital disease in dogs. J. Am. Vet. Med. Assoc. 230, 671-680.

Boston, S.E. 2010. Craniectomy and orbitectomy in dogs and cats. Can. Vet. J. 51, 537-540.

Brenner, R.J. and Gordon, L.M. 2011. Malignant seeding following percutaneous breast biopsy: documentation with comprehensive imaging and clinical implications. Breast J. 17, 651-656.

Collins, S.P., Matheson, J.S., Hamor, R.E., Mitchell, M.A., Labelle, A.L. and O'Brien, R.T. 2013. Comparison of the diagnostic quality of computed tomography images of normal ocular and orbital structures acquired with and without the use of general anesthesia in the cat. Vet. Ophthalmol. $16,352-358$.

Constantin, A., Brisson, M.L., Kwan, J. and Proulx, F. 2010. Percutaneous US-guided Renal Biopsy: A Retrospective Study Comparing the 16-gauge End-cut and 14-gauge Side-notch Needles. J. Vasc. Interv. Radiol. 21, 357-361.

Diaz, L.K., Wiley, E.L. and Venta, L.A. 1991. Are malignant cells displaced by large-gauge needle core biopsy of the breast?. Am. J. Roentgenol. 173, 1303-1313.

Featherstone, H.J. and Heinrich, C.L. 2013. Ophthalmic examination and diagnostics, part 1: the eye examination and diagnostic procedures. In Veterinary Ophthalmology, Eds., Gelatt K.N., Gilger, B.C. and Kern, T.J., MI: Wiley-Blackwell, pp: 540-541.

Finger, P.T. 2014. Minimally invasive anterior orbitotomy biopsy: finger's aspiration cutter technique (FACT). Eur. Ophthalmol. 22, 309-315.

Gelatt, K.N. and Withley, R.D. 2011. Surgery of the orbit. In Veterinary Ophthalmic Surgery, Eds., Gelatt, K.N. and Gelatt, J.P., MI: Elsevier, pp: 51-88.

Gilger, B.C., Withley, R.D. and McLaughlin, S.A. 1994. Modified lateral orbitotomy for removal of orbital neoplasms in two dogs. Vet. Surg. 23, 53-58.

Gupta, S., Sood, B., Gulati, M., Takhtani, D., Bapuraj, R., Khandelwal, N., Singh, U., Rajwanshi, A., Gupta, S. and Suri, S. 1999. Orbital mass lesions: US-guided fine-needle aspiration biopsy-experience in 37 patients. Radiol. 213, 568-572.

Hakannsson, N.W. and Hakannsson, B.W. 2010. Transfrontal orbitotomy in the dog: an adaptable three-step approach to the orbit. Vet. Ophthalmol. 13, 377-383.

Hendrix, D.V. and Gelatt, K.N. 2000. Diagnosis, treatment and outcome of orbital neoplasia in dogs: a retrospective study of 44 cases. J. Small. Anim. Pract. 41, 105-108.

Héran, F., Bergès, O., Blustajn, J., Boucenna, M., Charbonneau, F., Koskas, F., Lafitte, F., Nau, E., Roux, P., Sadik, J.C., Savatovsky, J. and 
Williams, M. 2014. Tumor pathology of the orbit. Diagn. Interv. Imaging 95, 933-944.

Kato, Y., Notake, H., Kimura, J., Murakami, M., Hirata, A., Saki, H. and Yanai, T. 2012. Orbital embryonal rhabdomyosarcoma with metastasis in a young dog. J. Comp. Pathol. 147, 191-194.

Klopfleisch, R., Sperling, C., Kershaw, O. and Gruber, A.D. 2011. Does the taking of biopsies affect the metastatic potential of tumours? A systematic review of reports on veterinary and human cases and animal models. Vet. J. 190, 31-42.

LeCouteur, R.A., Fike, J.R., Scagliotti, R.H. and Cann, C.E. 1982. Computed tomography of orbital tumors in the dog. J. Am. Vet. Med. Assoc. 180, 910-913.

Lederer, K., Ludewig, E., Hechinger, H., Parry, A.T., Lamb, C.R. and Kneissl, S. 2015. Differentiation between inflammatory and neoplastic orbital conditions based on computed tomographic signs. Vet. Ophthalmol. 18, 271-275.

Mason, D.R., Lamb, C.R. and McLellan, G.J. 2001. Ultrasonographic findings in 50 dogs with retrobulbar disease. J. Am. Anim. Hosp. Assoc. 37, 557-562.

Nair, L.K. and Sankar, S. 2014. Role of fine needle aspiration cytology in the diagnosis of orbital masses: a study of 41 cases. J. Cytol. 31, 87-90.

Nyland, T.G., Wallack, S.T. and Wisner, E.R. 2002. Needle-tract implantation following US-guided fine-needle aspiration biopsy of transitional cell carcinoma of the bladder, urethra, and prostate. Vet. Radiol. Ultrasound 43, 50-53.

Orlandi, D., Sconfienza, L.M., Lacelli, F., Bertolotto, M., Sola, S., Mauri, G., Savarino, E. and Serafini, G. 2013. Ultrasound-guided core-needle biopsy of extra-ocular orbital lesions. Europ. Radiol. 23, 1919-1924.

Ortiz, O., Bastug, D. and Ellis, B. 1996. CT-Guided Percutaneous Lateral Suprazygomatic Approach for Posterior Orbital Wall Biopsy. Skull Base Surg. 6, 249-251.
Penninck, D., Daniel, G.B., Brawer, R. and Tidwell, A.S. 2001. Cross-sectional imaging techniques in veterinary ophthalmology. Clin. Tech. Small Anim. Pract.16, 22-39.

Phillips, G. and Schneider, M. 1981. Ultrasonically guided percutaneous fine needle aspiration biopsy of solid masses. Cardiovasc. Intervent. Radiol. 4, 33-38.

Slatter, D.H. and Abdelbaki, Y. 1979. Lateral orbitotomy by zygomatic arch resection in the dog. J. Am. Vet. Med. Assoc. 175, 1179-1182.

Spiess, B.M. and Pot, S.A. 2013. Diseases and surgery of the canine orbit. In Veterinary Ophthalmology, Eds., Gelatt K.N., Gilger, B.C. and Kern, T.J., MI: Wiley-Blackwell, pp: 793-831.

Tani, E., Seregard, S., Rupp, G., Soderlind, V. and Skoog, L. 2006. Fine-needle aspiration cytology and immunocytochemistry of orbital masses. Diagn. Cytopathol. 34, 1-5.

Van der Woerdt, A. 2008. Orbital inflammatory disease and pseudotumor in dogs and cats. Vet. Clin. North. Am. Small Anim. Pract. 38, 389-401.

Vignoli, M., Rossi, F., Chierici, C., Terragni, R., De Lorenzi, D., Stanga, M. and Olivero, D. 2007. Needle tract implantation after fine needle aspiration biopsy (FNAB) of transitional cell carcinoma of the urinary bladder and adenocarcinoma of the lung. Schweiz Arch. Tierheilkd. 149, 314-318.

Wang, A.L., Ledbetter, E.C. and Kern, T.J. 2009. Orbital abscess bacterial isolates and in vitro antimicrobial susceptibility patterns in dogs and cats. Vet. Ophthalmol. 12, 91-96.

Woodcock, M.B. and Morgan, M.R.C. 1998. Ultrasound-guided Tru-cut biopsy of the breast. Ann. R. Coll. Surg. Engl. 80, 253-256.

Yarovoy,A., Bulgakova, E.S., Shatskikh,A.V., Uzunyan, D.G., Kleyankina, S.S. and Golubeva, O.V. 2013. CORE needle biopsy of orbital tumors. Graefe's Arch. Clin. Exp. Ophthalmol. 251, 2057-2061. 